\title{
Early Bacterial Cultures from Open Fractures - Differences Before and After Debridement
}

\author{
Fred Chuma Sitati ${ }^{1}$, Philip Ogutu Mosi ${ }^{2}$, Joseph Cege Mwangi ${ }^{1}$ \\ 1. School of Medicine, University of Nairobi, Kenya \\ 2. Voi County Hospital, Kenya
}

Correspondence to: Dr. Fred Sitati, P.O Box 895-00200 Nairobi, Kenya. Email: fredsitati@yahoo.com

\begin{abstract}
Background: The pattern of organisms found in open fractures is important in the selection of antibiotics for prophylaxis and empirical treatment. So far, there is paucity of data on local patterns of bacteria contaminating and infecting open fractures and their antibiotic susceptibility profile. Objective: To describe the pattern and antibiotic susceptibility of bacterial isolates obtained within 24 hours of injury from open fractures and to compare these to bacterial isolates from fractures that subsequently develop infection. Methods:A prospective study of 98 patients with open fractures seen at Kenyatta National Hospital (KNH) within 24 hours of injury between November 2015 and March 2016 was conducted. Swabs for culture and sensitivity were taken from the wounds and antibiotics initiated.Surgical debridement was subsequently carried out witha 14 day follow up for wound infection. Wounds thatbecame infected were sampled for cultures
\end{abstract}

\section{Introduction}

Up to $70 \%$ of open fractures are contaminated at the time of injury mostly by organisms from the skin and the environment (1). Contamination may also occur in the course of treatment. A few studies suggest that organisms isolated from wounds pre-debridement are similar to those isolated from wounds that get infected $(2,3)$. On the contrary, others find no association and indicate that infections are largely from nosocomial organisms $(4,5)$. These bacterial patterns are important in developing antibiotic protocols for prophylaxis and empirical treatment. Local studies on these patterns are lacking. The aim of this study was to describe the pattern of pre-debridement bacterial cultures and antibiotic susceptibility from open fractures within24 hours of injury at Kenyatta National Hospital (KNH).
And sensitivity. Results: Fifty-one (52.2\%) pre debridement wound samples had positive cultures. Staphylococcus aureus and (Coagulase Negative) CON Staphylococci were the predominant gram positive isolates. At 14 days' follow-up, the infection rate was $58.9 \%$. The predominant gram positive isolate was Staphylococcus aureus. Only $5.7 \%$ of the predebridement isolates were similar to the post debridement isolates. Conclusions: The contaminating organisms are not similar to the organisms isolated from injuries that later develop infection.

Key words: Open fracture, Antibiotics, Infection, Bacterial cultures.

Ann Afr Surg. 2017; 14(2):66-70

DOI:http://dx.doi.org/10.4314/aas.v14i2.3

(C) 2017 Author. This work is licensed under the Creative Commons Attribution 4.0 International License.

\section{Methods}

This was a prospective longitudinal study conducted over a 5-month period between November2015 and March 2016. Convenience sampling of patients above 18 years presenting within 24 hours of injury at the outpatient and accident and emergency (A\&E) departments of $\mathrm{KNH}$ with open limb fractures was carried outdone. KNH is Kenya's national tertiary, teaching and referral hospital with a 2000 bed capacity. We excluded patients who presented more than 24 hours after injury, had antiseptic wound dressing, had received antibiotics, presented with contamination from the gastrointestinal or genitourinary injuries or were known to have diabetes, peripheral vascular disease or immunosuppression. Informed consent was obtained 
from all participants and confidentiality upheld. A sample was collected from the wound as per Levine's technique (6). We collected samples from Accident and Emergency (A\&E) within 24 hours as per protocol and not debridement samples in theatre because most cases arrived in theatre two to three days after injury. The collected sample was transported to the laboratory in Amies or Stuart transport media. Wounds were covered by sterile gauze pending debridement. Participants had antibiotic prophylaxis initiated at the A\&E as per the British Orthopedic Association/British Association of Plastic, Reconstructive and Aesthetic Surgeons (BOA/BAPRAS) protocol (7). This is the usual protocol at $\mathrm{KNH}$. Cefuroxime 1.5 grams was administered parentally in the outpatient department and continued 8 hourly until first debridement. During the initial debridement, cefuroxime $1.5 \mathrm{~g}$ and gentamicin $(1.5 \mathrm{mg} / \mathrm{kg})$ was administered and continued 8 hourly until soft tissue closure or for a maximum of 72 hours, whichever occurred earlier. Surgical debridement was performed by the orthopedic resident on call. Fracture stabilization was by either external fixator for Gustillo II and III open fractures or plaster of Paris back slab or cast with a window for Gustillo I open fractures. Subsequently patients were followed up for a maximum period of 14 days, and development of wound infection assessed using the ASEPSIS score (8). Those with a score of above 20 had swabs taken as per Levine's technique described above (6).Samples taken pre-debridement and those that got infected were incubated in Sheep Blood agar and MacConkey agar at 35 to 37 degrees for 16 to 18 hours in aerobic atmosphere. The VITEK 2 automated system was used to identify positive cultures and determine antibiotic susceptibility (9-11). A questionnaire was administered to the patient by the principal investigator or the research assistant. The questionnaire collected data on the patient's age, gender, anatomical site of injury, mechanism of injury, Gustillo and Anderson classification of the injury, duration of time between injury and sample collection, duration of time between injury and debridement, duration to wound closure.

Data collected was then entered into a computer and analyzed using SPSS Version 17. A P-value of $<0.05$ was considered significant. Ethical Approval to conduct the study was sought and obtained from the Kenyatta National Hospital, Ethics and Research Committee.

\section{Results}

In the study period, 98 eligible subjects were recruited. Eighty $(83.6 \%)$ were male and $18(16.3 \%)$ females. Their average age was 30.5 years SD (10.53) with a range of $4-71$ years. Eighteen $(18.36 \%)$ of the fractures were Gustillo I, 38 (39.8\%) grade II, 22 (22.4.0\%) grade IIIA and 19 (19.4\%) grade IIIB. Most fractures involved the tibia/fibula (43.9\%). The average time to sample collection was 12.6 hours SD (3.5) with a range of 4 to 22 hours. Thirty-one (31.6\%) of the subjects were motorcycle occupants. Pedestrians $(20.4 \%)$ constituted the second largest group. There were $51(52.2 \%)$ positive cultures. Seven cultures had three organisms isolated while twelve had two isolates. Out of the positive cultures $55.8 \%$ were gram negative while $44.15 \%$ were gram positive. Staphylococcus aureus $(22.07 \%)$ and the Coagulase negative staphylococci $(19.5 \%)$ were the most common gram positive isolates while Pseudomonas aeruginosa $(11.7 \%)$ and Klebsiella spp. (10.4\%) were the most common gram negative isolates (See Table 1).

Table 1: Number of Organisms Isolated from PreDebridement and post -Debridement Swabs

\begin{tabular}{|l|c|c|}
\hline \multicolumn{1}{|c|}{ Bacteria } & $\begin{array}{c}\text { Pre- } \\
\text { Debridement }\end{array}$ & $\begin{array}{l}\text { Post - } \\
\text { Debridement }\end{array}$ \\
\hline Staph aureus & 15 & 12 \\
\hline CON Staphylococci & 14 & 5 \\
\hline Pseudomonas spp. & 9 & 10 \\
\hline Escherichia coli & 4 & 4 \\
\hline Klebsiella spp. & 7 & 5 \\
\hline Serratia spp. & 4 & 2 \\
\hline Actinetobacter spp. & 6 & 4 \\
\hline Morganella spp. & 4 & 2 \\
\hline Proteus spp. & 4 & 1 \\
\hline Provideciastuarti & 1 & 0 \\
\hline Raulotellaplanticola & 1 & 0 \\
\hline Strep agalactiae & 1 & 0 \\
\hline Streptococcus pyogenes & 1 & 0 \\
\hline
\end{tabular}

CON - Coagulase negative; Spp - species; Staph - Staphylococci

\section{Antibiotic Susceptibility Patterns of Cultures} Susceptibility of gram positive organisms to Vancomycin, Meropenem, and Teicoplanin was over $90 \%$ with no resistance to Linezolid. Susceptibility to 
Cefuroxime and Ceftriaxone was $83.3 \%$ and $82.6 \%$ respectively. There was high resistance to Tetracycline and the fluoroquinolones (Figure 1).

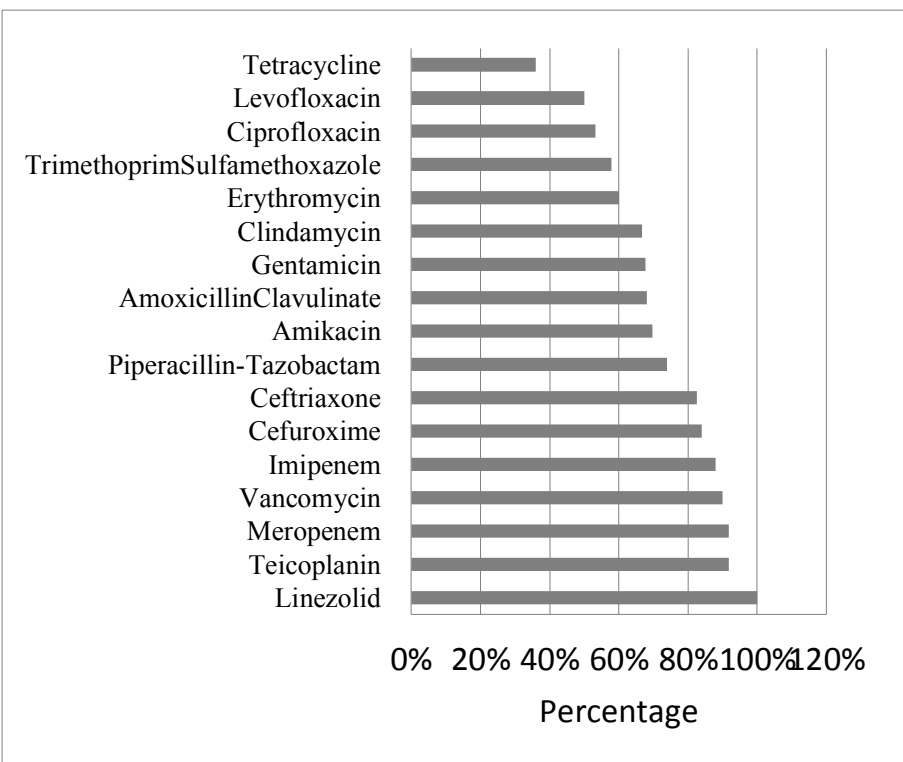

Figure 1: Antibiotic susceptibility of gram positive isolates from pre-debridement wound samples

None of the gram negative organisms isolated showed resistance to linezolid or Teicoplanin. However, they had high resistance to Tetracycline and Amoxicillin/Clavulanic acid (27\% and 22.3\%). Gentamycin and Amikacin had $87.5 \%$ and $91 \%$ susceptibility while cefuroxime had $47.6 \%$ susceptibility (See Figure 2)

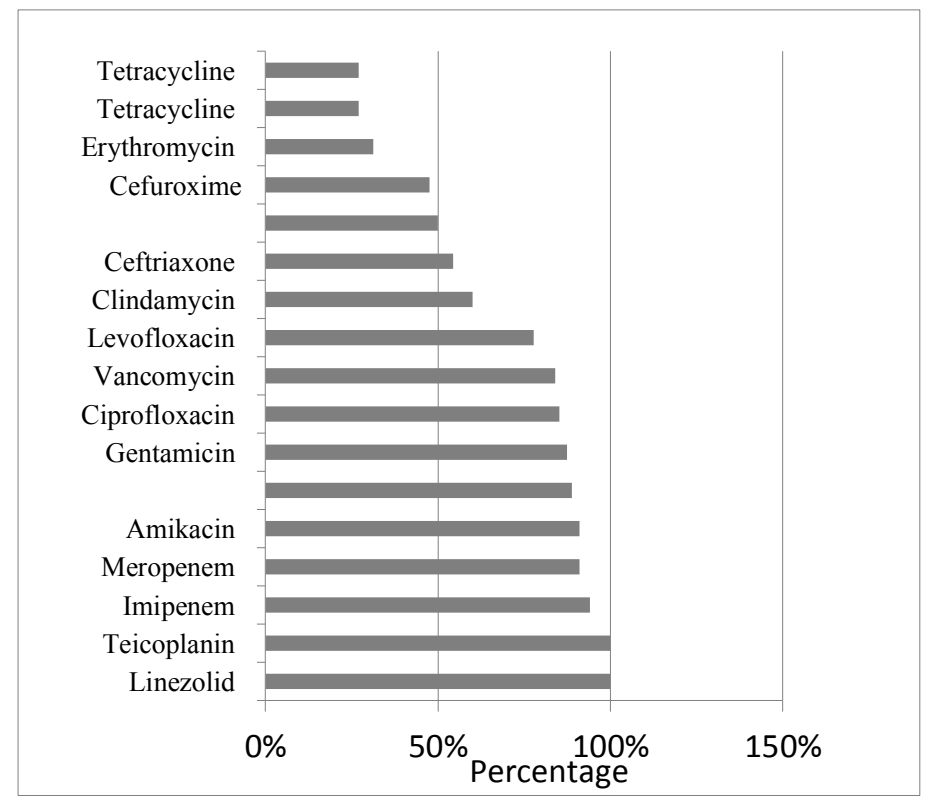

Figure 2: Antibiotic susceptibility of gram negative isolates from pre-debridement wound samples

\section{Injury Characteristics versus Rate of Positive Culture}

The median duration from time of injury to debridement in the positive and the negative culture groups was 12 and 7 hours respectively; $(\mathrm{p}=$ 0.76).There was no statistically significant difference between culture positivity and the mechanism of injury $(\mathrm{p}=0.71)$. A chi-square test of linear trend was performed to determine whether the severity in the Gustillo and Anderson grade was related to the outcome of the culture results. $X^{2}(1, \mathrm{~N}=98)=13.25$, $\mathrm{p}=0.07$. The rate of culture positivity increased with the severity of the Gustillo classification. There was no correlation between the type of organism cultured and the mode of injury $(p=0.56)$. There was a statistically significant trend with the patients presenting Grade IIIB being more likely to have both gram positive and negative organisms $(\mathrm{p}=0.01)$.

\section{Post-debridement Cultures}

The infection rate in this study was $58.9 \%$ (58 wounds). The number of positive cultures from infected wounds was 24 (43.7\%). Fifteen samples cultured multiple organisms. Eleven organisms were isolated. The predominant gram positive isolate was Staphylococcus aureus (25\%) while Pseudomonas spp. (20.8\%) and Klebsiella spp. (10.4\%) were the major gram negative isolates (See Table 1).

\section{Pre-debridement vs. Post-debridement Isolates}

Out of the 51 pre-debridement cultures only $3(5.7 \%)$ had a similar species isolated from the postdebridement samples: One Actinobacterbaumanni and two Staphylococcus aureus. The odds ratio of a wound with a positive pre-debridement culture developing infection was 1.86 (95\% Confidence interval of 0.82 to 4.21).

\section{Discussion}

This study sets out to establish the current pattern of bacterial organisms contaminating fresh open fractures seen at $\mathrm{KNH}$ and to compare these to bacterial isolates from those that develop infection. Our study population had gender and age distribution comparable to similar studies in Nigeria (12) and India (13). Local studies on open tibia fractures have demonstrated similar distributions (14-16). These studies also had comparable distribution of fractures as per the Gustillo 
and Anderson score, with most injuries classified as Gustillo II. The delay in swab collection was largely due to delay in patients' arrival to hospital and delays in the A\&E unit (17). The rate of positive cultures is lower than the documented average from most studies done in Europe and America. Gustillo et al found 70\% cultures being positive, but this was due to the methodology used where results from two swabs - one at the Accident and Emergency and the second at the time of debridement were added together. (1). Patzakis et al had a positive culture rate of $65 \%$ but these included anaerobic cultures whereas in our study did not include anaerobic cultures (18). Some studies have shown anaerobic organisms to account for up to $30 \%$ of infections. Studies in Nigeria, Ethiopia and India had lower positive culture yields ranging between $25 \%$ to $41 \%(12,13,19,20)$. The inclusion of patients who had received antibiotics may explain the low isolation rates in a study at the Black Lion Hospital in Addis Ababa. Various culturing techniques employed in the different studies may also explain the wide variation in positive culture rates. Almost equal gram positive and negative organisms were isolated from the predebridement samples. This is comparable to results from most studies. A few studies, such as that by Yishak et al. have gram negative cultures predominating (20). Gupta et al had 25 positive cultures out of a sample of 100. All organisms were gram negative (13).

From this study, the severity of the Gustillo classification is associated with the likelihood of having a positive culture. The type of organism was not associated with the site of injury, duration from injury or age. Gustillo IIIB injuries were likely to have both gram positive and negative contaminants. Staphylococcus aureus (22.07\%) was the main isolate, while the isolation rate for CON was $19.5 \%$. This is comparable to results by Akinyoola et al. in Nigeria that found Staphylococcus aureus at $22.2 \%$ and CON at $21.4 \%(16)$. Susceptibility to the cephalosporins was high among gram positives. Gram negative organisms showed higher susceptibility to flouroquinolones and aminoglycoside, especially amikacin. This is consistent with recommendations by the B.O.A. /B.A.P.R. protocol on prophylaxis in open fractures (7). Resistance to tetracycline and erythromycin was high in both gram positive and negative organisms. Vancomycin, imipenem, meropenem, teicoplanin and Linezolid were highly effective across both gram negative and gram positive organisms. These drugs are however costly and are not be recommended for prophylaxis. Their use should be limited to treatment of severe infection to protect their efficacy. A microbiologist or infectious disease specialist should be consulted in such cases.

The low correlation between pre-debridement and post-debridement swabs is consistent with results from similar studies $(4,5)$. This could be explained in two ways:

The isolates from the infected wounds may be those that were not covered by the spectrum of the prophylactic antibiotics or the organisms responsible for infection may be nosocomial.

Some studies have contrary findings. Lawrence et al found that $73 \%$ of infections were from bacteria in the pre-debridement cultures (4). Only two organisms were responsible for the infections in this study Serratiaspp and E.coli. D'Souza et al had similar findings in a study of 108 compound fractures (2).

\section{Conclusion}

At least $52 \%$ of open fractures are contaminated in our setting with an equal distribution of gram negative and positive organisms. Resistance to tetracycline, erythromycin and amoxicillin-clavulanic acid is high in both pre-debridement and post- debridement groups. The contaminating organisms are not similar to the infecting organisms

\section{Limitations}

This study had a short follow up period as well as lack of anaerobic cultures. Our culture samples were obtained from the A\&E department unlike the gold standard which is a debridement sample taken in theatre.

\section{Recommendations}

Further research into the topic should be carried out with inclusion of anaerobic cultures and D.N.A. fingerprinting to determine strains. We further recommend the use of intravenous Cefuroxime and gentamycin as antibiotic prophylaxis at $\mathrm{KNH}$. This should be updated regularly through surveillance 
studies. The BOA/BAPR protocol should be adopted in the intervening period.

\section{References}

1. Gustillo RB, Anderson JT. Prevention of Infection in the Treatment of One Thousand and TwentyFive Open Fractures of Long Bones: Retrospective and Prospective Analyses. J Bone Join Surg Am. 1976; 58(4):453-8.

2. D'Souza A, Rajagopalan N, Amaravati RS. The Use of Qualitative Cultures for Detecting Infection in Open Tibial Fractures. J Orthop Surg (Hong Kong). 2008; 16(2):175-8.

3. Kreder HJ, Amstrong P. The Significance of Perioperative Cultures in Open Pediatric LowerExtremity Fractures. Clin Orthop Relat Res. 1994; 302:206-12.

4. Robinson D, On E, Hadas N, et al. Microbiologic Flora Contaminating Open Fractures: It's Significance in the Choice of Primary Antibiotic Agents and the Likelihood of Deep Wound Infection. J Orthop Trauma. 1989; 3(4): 283-6.

5. Carsenti-Etesse H, Doyon F, Desplaces N, et al. Epidemiology of Bacterial Infection during Management of Open Leg Fractures. Eur J Clin Microbiol Infect Dis. 1999; 18(5):315-23.

6. Kallstrom G. Are Quantitative Bacterial Wound Cultures Useful? J. Clin. Microbiol. 2014; 52(8):2753-6.

7. Nanchahal J, Nayagam S, Khan U, et al. Standards for the Management of Open Fractures of the Lower Limb. London: Royal Society of Medicine Press Ltd; 2009

8. Wilson AP, Treasure T, Sturridge MF, et al. A scoring method (ASEPSIS) for postoperative wound infections for use in clinical trials of antibiotic prophylaxis. Lancet. 1986 8; 1(8476):311-3.

9. Becherucci V, Gentile F, Ceccantini R, et al. Validation of Rapid Sterility Test with BacT/ALERT 3D on Human Mesenchymal Stem Cells as Cell Therapy Product Inside an Hospital Cell Factory. IJOMAS. 2016; 2(4):13-21.

10. Romero-Gómez MP, Gómez-Gil R, Paño-Pardo $\mathrm{JR}$, et al. Identification and Susceptibility Testing of Microorganism by Direct Inoculation from Positive Blood Culture Bottles by Combining
MALDI-TOF and Vitek-2 Compact is Rapid and Effective. J. Infect. 2012; 65(6):513-20.

11. Chen JR, Lee SY, Yang BH, et al. Rapid Identification and Susceptibility Testing Using the Vitek 2 System Using Culture Fluids from Positive BacT/ALERT Blood Cultures. J Microbiol Immunol Infect. 2008; 41(3): 259-64.

12. Akinyoola A, Ako-Nai A, Dosumu O, et al. Microbial Isolates in Early Swabs of Open Musculoskeletal Injuries. Niger Postgr Med J. 2006; 13(3):176-81.

13. Gupta S, Saini N, Sharma R, et al. A Comparative Study of Efficacy of Pre and Post Debridement Cultures in Open Fractures. Internet J Orthop Surg. 2012; 19(3).

14. Admani A. The Effect of Delay in Initial Debridement on the Rate of Infection in Open Fractures of Tibial Shaft at Kenyatta National Hospital - a Prospective Study. (Unpublished Masters Dissertation). University of Nairobi; 2012

15. Mogire J. The Management of Fresh Traumatic Open Tibial Shaft Fractures at Kenyatta National Hospital. (Unpublished Masters Dissertation). University of Nairobi; 1994.

16. Ondari J. Comparative Study of 24-Hour versus Five-Day Prophylactic Antibiotic Use in Gustillo II Open Tibia Fractures at Kenyatta National Hospital. (Unpublished Masters Dissertation) University of Nairobi; 2015.

17. Saidi H. Initial Injury Care in Nairobi, Kenya: A Call for Trauma Care Regionalization. East Afr Med J. 2003; 80(9):480-3.

18. Patzakis MJ, Wilkins J, Moore TM. Considerations in Reducing the Infection Rate in Open Tibial Fractures. Clin Orthop Relat Res. 1983; (178):3641.

19. Ikem IC, Oginni LM, Bamgboye EA, et al. The Bacteriology of Open Fractures in Ile-Ife, Nigeria. Niger J Med. 2004; 13(4):359-65.

20. Yishak A and Biruk L. Microbial Susceptibility of Bacteria Isolated from Open Fracture Wounds Presenting to the ER of Black-Lion Hospital, Addis Ababa University, Ethiopia. African J Microbiol Res. 2009;3(12):939-5 